\title{
Direct detection of a magnetic field in the photosphere of the single M giant EK Bootis ${ }^{\star}$
}

\section{How common is magnetic activity among $M$ giants?}

\author{
R. Konstantinova-Antova ${ }^{1,2}$, M. Aurière ${ }^{2}$, C. Charbonnel ${ }^{3,2}$, N. A. Drake ${ }^{4}$, K.-P. Schröder ${ }^{5}$, I. Stateva ${ }^{1}$, E. Alecian ${ }^{6}$, \\ P. Petit ${ }^{2}$, and R. Cabanac ${ }^{2}$
}

1 Institute of Astronomy, Bulgarian Academy of Sciences, 72 Tsarigradsko shose, 1784 Sofia, Bulgaria e-mail: renada@astro.bas.bg

${ }^{2}$ Laboratoire d'Astrophysique de Toulouse-Tarbes, Université de Toulouse, CNRS, Observatoire Midi Pyrénés, 57 Avenue d'Azereix, 65008 Tarbes, France

3 Observatoire Astronomique de l'Université de Genève, 51 Chemin des Maillettes, 1290 Versoix, Switzerland

4 Sobolev Astronomical Institute, St. Petersburg State University, Universitetski pr. 28, St. Petersburg 198504, Russia

5 Departamento de Astronomia, Universidad de Guanajuato, A.P. 144, C.P. 36000, GTO, Mexico

${ }^{6}$ Laboratoire d'Astrophysique de Grenoble, Université Joseph Fourier-CNRS, BP 53, 38041 Grenoble Cedex 9, France

Received 25 March 2010 / Accepted 1 September 2010

\section{ABSTRACT}

\begin{abstract}
Aims. We study the fast rotating M 5 giant EK Boo by means of spectropolarimetry to obtain direct and simultaneous measurements of both the magnetic field and activity indicators, in order to infer the origin of the activity in this fairly evolved giant.

Methods. We used the new spectropolarimeter NARVAL at the Bernard Lyot Telescope (Observatoire du Pic du Midi, France) to obtain a series of Stokes $I$ and Stokes $V$ profiles for EK Boo. Using the least square deconvolution (LSD) technique we were able to detect the Zeeman signature of the magnetic field. We measured its longitudinal component by means of the averaged Stokes $V$ and Stokes $I$ profiles. The spectra also permitted us to monitor the $\mathrm{Ca} I \mathrm{II} \& \mathrm{H}$ chromospheric emission lines, which are well known as indicators of stellar magnetic activity.

Results. From ten observations obtained between April 2008 and March 2009, we deduce that EK Boo has a magnetic field, which varied in the range of -0.1 to $-8 \mathrm{G}$. On March 13, 2009, a complex structure of Stokes $V$ was observed, which might indicate a dynamo. We also determined the initial mass and evolutionary stage of EK Boo, based on up-to-date stellar evolution tracks. The initial mass is in the range of 2.0-3.6 $M_{\odot}$, and EK Boo is either on the asymptotic giant branch (AGB), at the onset of the thermal pulse phase, or at the tip of the first (or red) giant branch (RGB). The fast rotation and activity of EK Boo might be explained by angular momentum dredge-up from the interior, or by the merging of a binary.

In addition, we observed eight other $\mathrm{M}$ giants, which are known as X-ray emitters, or to be rotating fast for their class. For one of these, $\beta$ And, presumably also an AGB star, we have a marginal detection of magnetic field, and a longitudinal component $B_{1}$ of about $1 \mathrm{G}$ was measured. More observations like this will answer the question whether EK Boo is a special case, or whether magnetic activity is, rather, more common among $\mathrm{M}$ giants than expected.
\end{abstract}

Key words. dynamo - magnetic fields - instrumentation: polarimeters - techniques: polarimetric - starts: activity - stars: evolution

\section{Introduction}

There are already measurements of magnetic fields in single $G$ and $\mathrm{K}$ giants (Konstantinova-Antova et al. 2008a; Aurière et al. 2008; Konstantinova-Antova et al. 2009), but M giants have not yet been studied in this respect. Despite theoretical predictions of possible dynamo operation on the asymptotic giant branch (AGB) (Blackman et al. 2001; Soker \& Zoabi 2002; Nordhaus et al. 2008), actual evidence of magnetic activity in such evolved stars is sparse and indirect (Hünsch et al. 1998; Karovska et al. 2005; Herpin et al. 2006). Hence, we here present a study of the variable magnetic field of the single $\mathrm{M}$ giant EK Boo. This work is a part of a programme, that aims to detect magnetic fields in evolved, single late-type stars (Konstantinova-Antova et al. 2008a)

* Based on data obtained using the Télescope Bernard Lyot at Observatoire du Pic du Midi, CNRS and Université de Toulouse, France.
The source EK Boo = HD 130144 is a 6th magnitude M 5 giant and a semiregular variable star (Kholopov et al. 1998). This evolved giant attracted our interest by its unusual X-ray luminosity $L_{\mathrm{x}}$, which is higher than $10^{30} \mathrm{erg} \mathrm{s}^{-1}$ (Hünsch et al. 1998). A further study revealed its variability of $L_{\mathrm{x}}$, as well as its optical activity indicators $\mathrm{Ca}$ II $\mathrm{K} \& \mathrm{H}$ and $\mathrm{H}_{\alpha}$ (Hunsch et al. 2004). At the same time, EK Boo is considered to be a single star (Famaey et al. 2009) with a projected rotation velocity of $v \sin i=11 \mathrm{~km} \mathrm{~s}^{-1}$ (Hünsch et al. 2004). A direct detection of its magnetic field was first reported by Konstantinova-Antova et al. (2009). To our knowledge, this was the first direct detection of a magnetic field in a single $\mathrm{M}$ giant. In the work we present here, we study the variability of the magnetic field and activity of EK Boo, its chemical composition and evolutionary stage, and we discuss possible reasons for a dynamo operation in such an evolved star. To see if this magnetically active $M$ giant is a special case, or magnetic activity is common in such stars, we also present first magnetic field measurements obtained for other M giants. 
Table 1. Journal of observations of EK Boo.

\begin{tabular}{lcccccc}
\hline \hline Date & $\begin{array}{c}\text { HJD } \\
2450000+\end{array}$ & $\begin{array}{c}\text { Exp. T. } \\
\text { s }\end{array}$ & Det. & $\begin{array}{c}B_{1} \\
\mathrm{G}\end{array}$ & $\begin{array}{c}\sigma \\
\mathrm{G}\end{array}$ & Ca II K \\
\hline 03 Apr. 08 & 4560.50 & 800 & $\mathrm{DD}$ & -6.7 & 1.8 & 0.39 \\
04 Apr. 08 & 4561.53 & 1600 & DD & -3.1 & 0.5 & 0.39 \\
05 Apr. 08 & 4562.54 & 1600 & DD & -5.0 & 0.5 & 0.40 \\
06 Apr. 08 & 4563.51 & 1600 & DD & -4.7 & 0.7 & 0.36 \\
20 Dec. 08 & 4821.74 & 2400 & nd & -0.1 & 0.6 & 0.46 \\
21 Dec. 08 & 4822.74 & 2800 & nd & -0.5 & 0.6 & 0.49 \\
25 Feb. 09 & 4888.6 & 3200 & DD & -0.3 & 0.4 & 0.57 \\
09 Mar. 09 & 4900.57 & 3200 & DD & -3.8 & 0.6 & 0.55 \\
13 Mar. 09 & 4904.68 & 3200 & DD & -4.6 & 0.4 & 0.56 \\
18 Mar. 09 & 4909.55 & 3200 & DD & -8.1 & 0.6 & 0.57 \\
\hline
\end{tabular}

Notes. Individual columns correspond to Dates, HJD, total exposure time, Zeeman detection ( $\mathrm{DD}=$ definitive detection, $\mathrm{nd}=$ no detection $)$, $B_{1}$ values and its errors in $\mathrm{G}$, and $I(\mathrm{CaII} \mathrm{K}) / I(3950)$ for Ca II K emission.

\section{Observations and methods}

The observations of EK Boo were carried out at the 2-m Bernard Lyot Telescope (TBL) of the Pic du Midi observatory, with NARVAL, a new generation spectropolarimeter (Aurière 2003). It is a copy of the instrument ESPaDOnS, which was installed at CFHT at the end of 2004 (Donati et al. 2006). NARVAL is a fiber-fed echelle spectrometer, able to cover the whole spectrum from $370 \mathrm{~nm}$ to $1000 \mathrm{~nm}$ in a single exposure. Forty orders are aligned on the CCD frame, separated by 2 cross-disperser prisms. We used NARVAL in polarimetric mode, with a spectral resolution of 65000 . Stokes $I$ (unpolarised) and Stokes $V$ (circular polarization) parameters were measured by means of four sub-exposures, between which the retarders, Fresnel rhombs, were rotated in order to exchange the beams in the instrument and to reduce spurious polarization signatures.

We observed EK Boo during four consecutive nights in April 2008, then on 20 and 21 December 2008, 25 February 2009, and during three nights in March 2009. For the extraction of the spectra we used Libre-ESpRIT (Donati et al. 1997), a fully automatic reduction package installed at TBL. For the Zeeman analysis, a least-squares deconvolution (LSD, Donati et al. 1997) was then applied to all spectra. We used a mask, which was calculated for solar abundance, an effective temperature of $3500 \mathrm{~K}, \log g=0.5$ and a microturbulence of $2.0 \mathrm{~km} \mathrm{~s}^{-1}$. These parameters are consistent with the spectral class and luminosity of EK Boo (Dyck et al. 1998). This method enabled us to average a total of about 12700 lines and to get Stokes $I$ and Stokes $V$ profiles. The null spectrum ( $N$ profile) given by the standard procedure (Donati et al. 1997) was also examined, but never showed any signal, confirming that the detected signatures in the $V$ profiles are not spurious. From the obtained mean Stokes $V$ for each night we computed the surface-averaged longitudinal magnetic field $B_{1}$ in G, using the first-order moment method (Donati et al. 1997; Rees \& Semel 1979).

At the same time, the magnetic activity of EK Boo was monitored by means of measurements of the relative intensity of the chromospheric activity indicator Ca II K. In the core of this emission line, the $S / N$ of our spectra exceeds 50 . Table 1 gives the journal of our observations of EK Boo, including the dates, Heliocentric Julian Day (HJD), total exposure time, detectionlevel from the LSD statistics (Donati et al. 1997), $B_{1}$ and its error (in G), and the relative intensity $I(\mathrm{CaII} \mathrm{K}) / I(3950)$ for the maximum of the $\mathrm{Ca}$ II $\mathrm{K}$ emission.

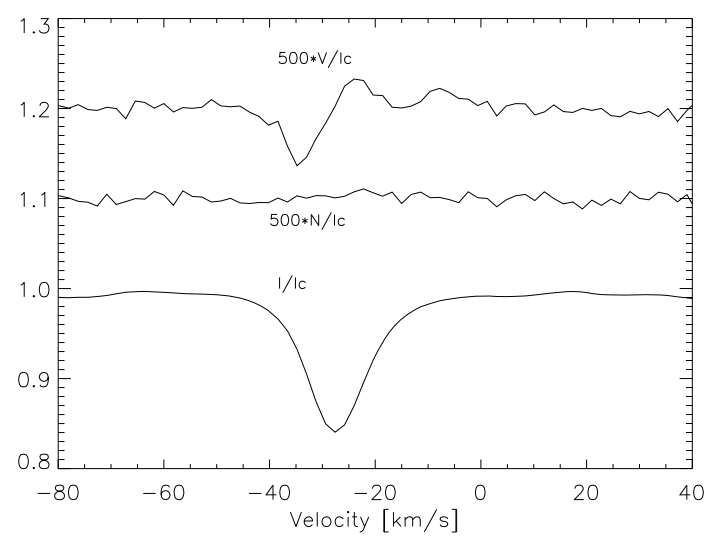

Fig. 1. LSD profiles of Stokes $I$, null polarization $N$ and Stokes $V$ (bottom, middle and upper curves) for EK Boo, as observed on 13 March 2009. For clarity, Stokes $V$ and $N$ profiles are enlarged 500 times, and the successive profiles are shifted vertically.

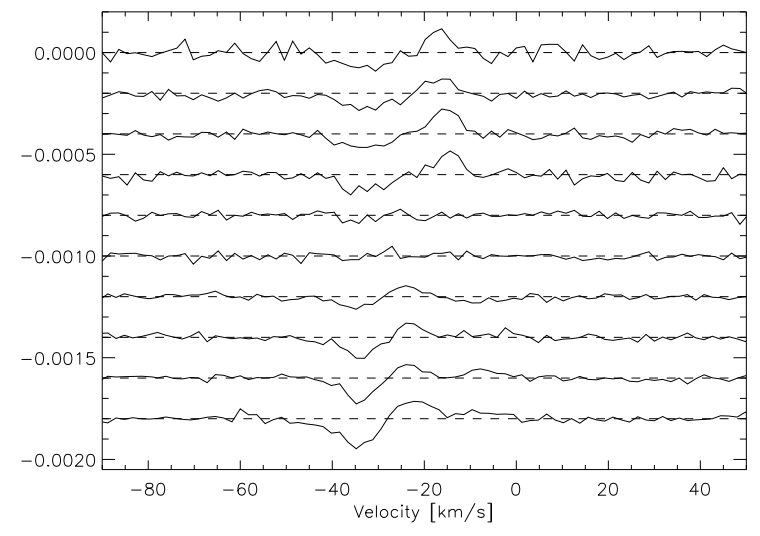

Fig. 2. LSD Stokes $V$ profiles for EK Boo for the dates listed in Table 1, from 03 Apr. 08 (top) to 18 Mar. 09 (bottom). The dashed lines illustrate the respective zero levels. The scale of the $Y$-axis is in units of $V / I_{\mathrm{c}}, I_{\mathrm{c}}$ is the intensity of the continuum. For clarity, successive profiles are shifted vertically.

\section{Observational results}

\subsection{Magnetic field and activity indicators of EK Boo}

A significant Stokes $V$ signal was detected at all times, except in December 2008. The measured $B_{1}$ varies from -0.1 to $-8 \mathrm{G}$. Figure 1 shows our best LSD profiles, obtained on 13 March 2009, under excellent sky conditions. Using the LSD statistics tool (Donati et al. 1997), the outstanding Stokes $V$ signature (upper profile) corresponds to a definitive detection (DD) with a $\chi^{2}$ of 4.7. The Stokes $V$ signal is well centred on the Stokes $I$ profile (bottom) and no signal at all is observed on the null polarization $N$ profile (middle). This observation shows a complex Stokes $V$ structure, maybe a result of a dynamo action. Figure 2 shows all observed Stokes $V$ profiles for EK Boo. A very weak signal was observed in the two nights of December 2008, and we did not obtain any significant Zeeman detection. Nevertheless, a definitive detection can be attained by averaging all spectra taken during these two nights. On 25 February 2009, the magnetic field was definitely detected again and the resulting $B_{1}$ is still close to 0 . This challenges our much higher measurements obtained in the four nights of April 2008, when the magnetic field of EK Boo did not present significant variations. And there appears a rapid increase of $B_{1}$ again in March 2009.

A significant $\mathrm{Ca}$ II $\mathrm{K} \& \mathrm{H}$ emission is observed in every night. The ratio $I / I(3950)$ of the K-line is given in Table 1 . In Fig. 3 

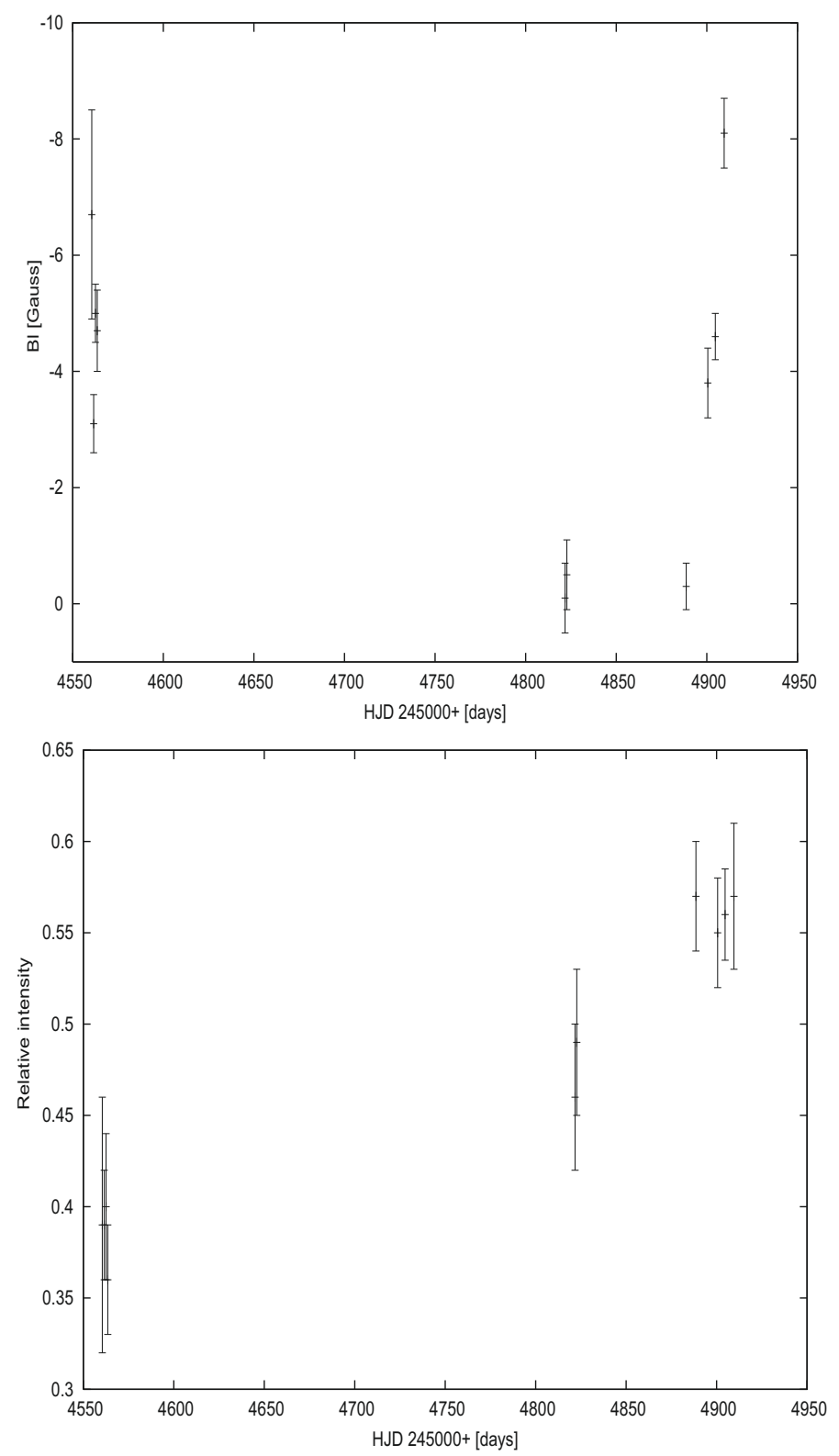

Fig. 3. Magnetic field measurements (upper panel) and chromospheric activity indicator Ca II K emission (lower panel) for EK Boo in the observed period. An inverse $Y$-axis is used for $B_{1}$ to illustrate the magnetic field.

it can be compared with the $B_{1}$ measurements. Both measurements are plotted over HJD. We can see that the Ca II K emission increases smoothly and monotonically during the period monitored here, apparently without any significant short-time variation. This behaviour is different from that of the Stokes $V$ profiles and $B_{1}$. For example, Ca II is at the weakest when the magnetic field is strong in the nights in April 2008. And it becomes stronger when Stokes $V$ and $B_{1}$ are weak on 20 and 21 December 2008. This suggest the existence of areas with opposite magnetic polarity on the surface of EK Boo: contributions of both polarities to the mean magnetic field can cancel each other when both are present on the facing side of the star, while adding up to a maximum Ca II emission line flux of the related chromospheric activity regions.

The Ca II K emission profiles sometimes feature a single peak, but on other occations they show a double-peak structure (see Fig. 4). Their peak asymmetry changes from $V / R>1$ on

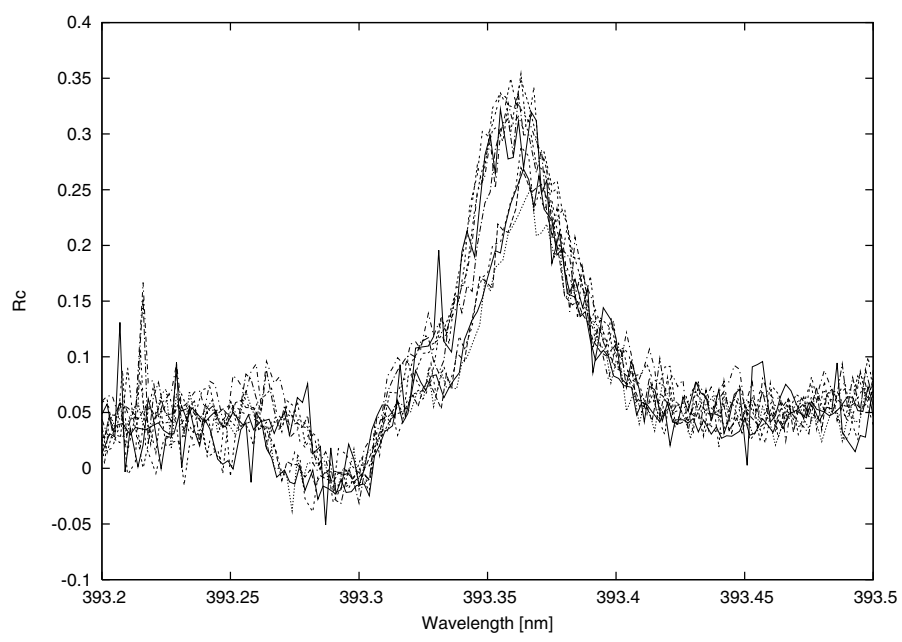

Fig. 4. Ca II K emission line profiles, as observed for EK Boo in the period of April 2008 to March 2009.

April 3, 2008, to $V / R<1$ on December 21, 2008, where $V / R$ is the ratio between the violet $(V)$ and red $(R)$ components of the double-peaked emission line. In the first case, this indicates vertical motions in the chromosphere, where the downward components appear stronger. This is typical for many red giants (Smith $\&$ Shetrone 2000). In the second case, $V / R<1$, upward motions and possible outflow dominate the asymmetry. Hence, the chromosphere of EK Boo seems to be very dynamical on timescales of months and years, with episodic outflows from its lower layers, where $\mathrm{Ca}$ II $\mathrm{K}$ and $\mathrm{H}$ emission lines are formed.

\subsection{Radial velocity, binarity, and pulsations}

Radial velocity measurements $(R V)$ for EK Boo have been carried out on the LSD Stokes $I$ profiles using Gaussian fits. The $R V$ accuracy of NARVAL is 20-30 $\mathrm{m} \mathrm{s}^{-1}$ (Moutou et al. 2007; Aurière et al. 2009). Variations of $R V$ larger than $1 \mathrm{~km} \mathrm{~s}^{-1}$ were observed on a time-scale of less than a month. But in averaging the $R V$ measurements for each month (April 2008, December 2008, February 2009, and March 2009), we find a standard deviation of only about half a $\mathrm{km} \mathrm{s}^{-1}$. This compares well with the values ( 0.36 and $0.65 \mathrm{~km} \mathrm{~s}^{-1}$ respectively) obtained by Hünsch et al. (2004) and Famaey et al. (2009) from measurements with CORAVEL (Udry et al. 1997). Famaey et al. (2009) included their EK Boo observations in two of their diagnosis plots and concluded that the star "appears amidst the non-binary M giants".

EK Boo is also known as a semi-regular variable (Kholopov et al. 1998). A larger photometric sample is available from Hipparcos data, and though EK Boo is among stars with large HIP photometric amplitudes (0.38 mag, Adelman 2001), no clear periodicity can be derived (Koen et al. 2002).

The average of our much more recent $R V$ measurement is shifted by about $5 \mathrm{~km} \mathrm{~s}^{-1}$ with respect to the average given in the literature (about $-27 \mathrm{~km} \mathrm{~s}^{-1}$ with respect to about $-22 \mathrm{~km} \mathrm{~s}^{-1}$ ). This difference is one order of magnitude larger than any shift obtained for other $M$ giants studied with NARVAL, even when observations were made during the same nights as EK Boo. Hence, this shift appears to be real, and it could be linked to pulsations, as discussed in the context of other semiregular variable stars (Lebzelter 1999; Lebzelter et al. 2000) or of multiple-mode asymptotic giant branch variable stars (Hinkle et al. 2002). 
In summary, our $R V$ measurements confirm that their variations on a time-scale of less than a month are of a magnitude of about $1 \mathrm{~km} \mathrm{~s}^{-1}$. These variations must be considered as significant with respect to NARVAL's accuracy (better than CORAVEL accuracy). The analysis by Famaey et al. (2009) favours an origin from pulsations, quite common in this type of stars. Furthermore, the shift in $R V$ between our observations and those in the literature is not due to binarity, although slow orbital motion might, a priory, be a possible explanation: Hünsch et al. (1998) already considered the possibility of active, faint companions in detail, when discussing the origin of detected X-ray emission in M-giants. But in the case of EK Boo, spectral signatures of a secondary have never been found. On the other hand, clearly a Zeeman detection with NARVAL could only be caused by a bright object, which would have been easily detected spectroscopically. Hence, the M giant itself must be magnetically active.

\subsection{Metallicity and atmospheric parameters determination}

We used our NARVAL spectrum of 13 March 2009 to derive the metallicity and other parameters of EK Boo. For this purpose, we measured equivalent widths of the Fe I and Ni I lines in the "window" 7400-7580 $\AA$, a spectral region relatively free of molecular absorption. Values for the respective oscillator strengths $\log g f$ were taken, if available, from Gurtovenko $\&$ Kostyk (1989). Otherwise, we used the values derived by Smith \& Lambert (1985) or those offered by the VALD database (Kupka et al. 2000).

We then used spherically symmetric, LTE, hydrostatic model atmospheres as computed with the MARCS code (Gustaffson et al. 2008). The CNO abundances in atmospheres of $\mathrm{M}$ giants are known to be modified through the first dredge-up (see, e.g., Charbonnel 1994; Boothroyd \& Sackmann 1999; and Sect. 4): ${ }^{12} \mathrm{C}$ is depleted and ${ }^{14} \mathrm{~N}$ is enhanced. We therefore used MARCS models for moderately $\mathrm{CN}$-cycled composition with $[\mathrm{C} / \mathrm{Fe}]=$ $-0.13,[\mathrm{~N} / \mathrm{Fe}]=+0.31$, and ${ }^{12} \mathrm{C} /{ }^{13} \mathrm{C}=20$. This chemical composition agrees with the abundance ratios measured in field $\mathrm{M}$ giants by Smith \& Lambert (1990): $[\mathrm{C} / \mathrm{Fe}]=-0.19 \pm 0.13$ and $[\mathrm{N} / \mathrm{Fe}]=+0.28 \pm 0.11$.

The stellar mass was taken to be $M=2 M_{\odot}$ (see Sect. 4). But as shown by Plez (1990) and García-Hernandez (2007), the temperature and pressure structures of model atmospheres are practically identical for models of $1 M_{\odot}$ and $10 M_{\odot}$ stars. The current version of the LTE line analysis and spectrum synthesis code MOOG (Sneden 1973) was used here.

The microturbulence velocity was determined from the Fe I lines by requiring that the derived abundances shall be independent of the equivalent width. The effective temperature was derived by requiring that the abundance calculated for the Fe I lines shall not show any dependence on excitation potential (Fig. 5). The atomic line list, measured equivalent widths, and derived abundances are listed in Table 2.

In the way described above, we derive an effective temperature of $T_{\text {eff }}=3400 \mathrm{~K}$ and a microturbulent velocity of $\xi=2.0 \mathrm{~km} \mathrm{~s}^{-1}$. In adopting these values, a surface gravity of $\log g=0.0$ (estimated from $M_{\text {bol }}, T_{\text {eff }}$ and the stellar masses obtained by comparison with evolutionary tracks, see Sect. 4 and Fig. 7), we then derived the following abundances: $\log \varepsilon(\mathrm{Fe})=$ $7.38 \pm 0.13, \log \varepsilon(\mathrm{Ni})=6.12 \pm 0.18$ or $[\mathrm{Fe} / \mathrm{H}]=-0.14 \pm 0.13$ and $[\mathrm{Ni} / \mathrm{H}]=-0.13 \pm 0.18^{1}$ where the solar abundances of $\mathrm{Fe}$ and

\footnotetext{
${ }^{1}$ We adopt here the usual spectroscopic notation $[\mathrm{X} / \mathrm{Fe}]=\log \left(N_{\mathrm{X}} /\right.$ $\left.N_{\mathrm{Fe}}\right)_{\star}-\log \left(N_{\mathrm{X}} / N_{\mathrm{Fe}}\right)_{\odot}$.
}
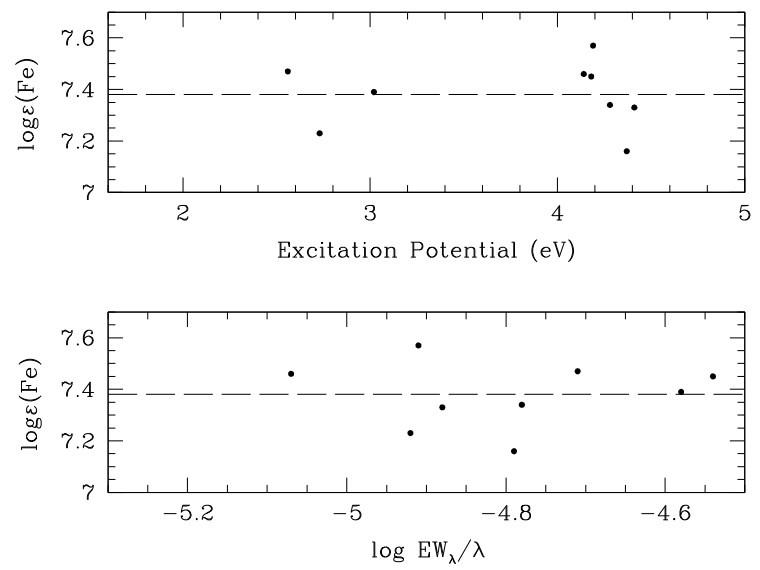

Fig. 5. Iron abundance as derived from individual Fe I lines, $\log \varepsilon(\mathrm{Fe})$, versus excitation potential and reduced equivalent width, $\log \left(W_{\lambda} / \lambda\right)$. An atmospheric model with $T_{\text {eff }}=3400 \mathrm{~K}, \log g=0.0$, and $\xi=$ $2.0 \mathrm{~km} \mathrm{~s}^{-1}$ was used.

Table 2. Observed Fe I and NiI lines, measured equivalent widths $(E W)$, and derived abundances.

\begin{tabular}{lccccc}
\hline \hline Element & $\lambda(\AA)$ & $\chi(\mathrm{eV})$ & \multicolumn{1}{c}{$\log g f$} & $E W(\mathrm{~m} \AA)$ & $\log \varepsilon(\mathrm{Fe})$ \\
\hline Fe I & 7443.022 & 4.19 & $-1.810^{1}$ & 91.2 & 7.57 \\
& 7461.520 & 2.56 & $-3.570^{1}$ & 145.7 & 7.47 \\
& 7498.530 & 4.14 & $-2.240^{1}$ & 63.3 & 7.46 \\
& 7507.266 & 4.41 & $-1.090^{1}$ & 99.0 & 7.33 \\
& 7511.019 & 4.18 & $0.099^{2}$ & 214.8 & 7.45 \\
& 7531.144 & 4.37 & $-0.610^{1}$ & 122.1 & 7.16 \\
& 7540.430 & 2.73 & $-3.870^{1}$ & 91.0 & 7.23 \\
& 7568.899 & 4.28 & $-0.890^{1}$ & 124.9 & 7.34 \\
Ni I & 7583.787 & 3.02 & $-1.930^{1}$ & 199.3 & 7.39 \\
& 7393.600 & 3.61 & $-0.100^{1}$ & 173.9 & 6.28 \\
& 7414.500 & 1.99 & $-1.970^{3}$ & 220.5 & 6.06 \\
& 7555.598 & 3.85 & $0.100^{1}$ & 137.8 & 5.90 \\
& 7574.043 & 3.83 & $-0.470^{1}$ & 126.9 & 6.26 \\
\hline
\end{tabular}

Notes. ${ }^{(1)}$ Gurtovenko \& Kostyk (1989); (2) VALD data base; ${ }^{(3)}$ Smith \& Lambert (1985).

Table 3. Abundance uncertainties for EK Boo.

\begin{tabular}{lcccccc}
\hline \hline Species & $\Delta T_{\text {eff }}$ & $\Delta T_{\text {eff }}$ & $\Delta \log g$ & $\Delta \log g$ & $\Delta \xi$ & $\Delta \xi$ \\
& $+100 \mathrm{~K}$ & $-100 \mathrm{~K}$ & +0.5 & -0.5 & +0.3 & -0.3 \\
\hline Fe I & -0.12 & +0.12 & +0.18 & -0.20 & -0.16 & +0.18 \\
Ni I & -0.11 & +0.11 & +0.19 & -0.20 & -0.21 & +0.25 \\
\hline
\end{tabular}

Notes. The second and third columns give the variation of the abundance that are caused by using a different $T_{\text {eff }}$, i.e. larger or smaller by $100 \mathrm{~K}$. The other columns refer to changes in $\log g$ and $\xi$ respectively.

Ni are from Anders \& Grevesse (1989). These values indicate a metallicity typical of $\mathrm{M}$ giants.

The accuracy of the above-derived metallicity of EK Boo depends on the uncertainties in the adopted atmospheric parameters. Spectral signatures of singly ionized elements are highly sensitive to the variations in $\log g$, and to a lesser extent to the variations of $T_{\text {eff. }}$. Small changes in the microturbulent velocity lead to larger changes in the derived abundances. Table 3 lists the abundance changes that are caused by individual changes in the various atmospheric parameters. In summary, we can state that the metallicity of EK Boo is comparable to the solar one. 


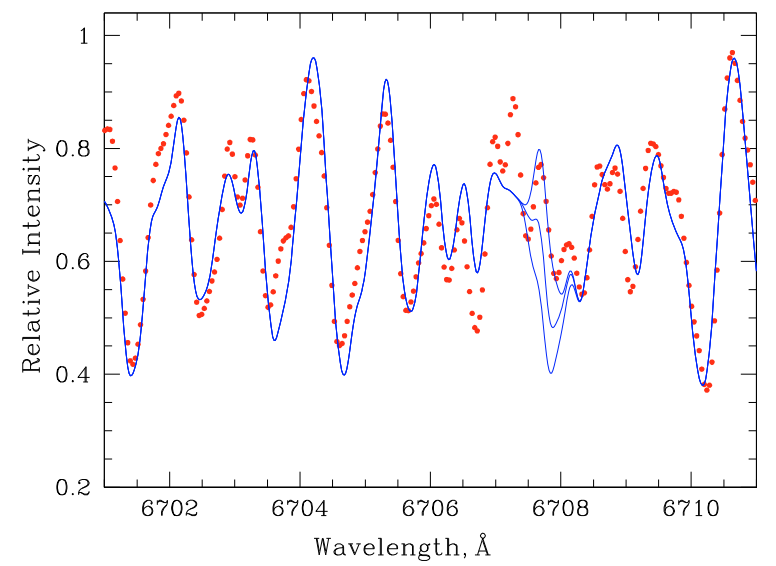

Fig. 6. Observed (dots) and synthetic (solid lines) spectra for EK Boo in the region around the Li I $6708 \AA$ resonance line, the observed spectrum is scaled in intensity for a good match. The synthetic spectra represent lithium abundances of $\log \varepsilon(\mathrm{Li})=-1.0,0.0$, and +1.0 .

\subsection{Lithium abundance}

In order to determine the lithium abundance of EK Boo, we used the Li I resonance doublet at $6707.8 \AA$. In this region, the spectrum is heavily blanketed by $\mathrm{TiO}$ molecular lines, especially by the $\gamma$ electronic transition. In our computations of synthetic spectra we used the TiO line list given by Plez (1998), restricted to ${ }^{48} \mathrm{TiO}$. In deed, when Plez et al. (1993) computed a restricted line list for the $\gamma$ system of $\mathrm{TiO}$ around the $6708 \AA \mathrm{Li}$ I line for all five isotopes of Ti from ${ }^{46} \mathrm{Ti}$ to ${ }^{50} \mathrm{Ti}$, they found that even if they included all isotopes to improve the match between calculated and observed spectra, this did not change the derived $\mathrm{Li}$ abundance.

Vacuum values of wavelengths $\left(\lambda_{\text {vac }}\right)$ were transformed to air wavelengths $\left(\lambda_{\text {air }}\right)$ according to the dispersion formula (see Birch \& Downs 1994; Morton 2000). For the dissociation energy of TiO, a value of $D_{0}=6.87 \mathrm{eV}$ was used. CN lines in the vicinity of the Li I doublet were included in our line list, even though the $\mathrm{CN}$ red system lines near $6700 \AA$ are very weak in M giants (Luck \& Lambert 1982). Wavelengths and oscillator strength for the individual hyperfine and isotopic components of the lithium lines were taken from Hobbs et al. (1999). A solar ${ }^{6} \mathrm{Li} /{ }^{7} \mathrm{Li}$ isotopic ratio $\left({ }^{6} \mathrm{Li} /{ }^{7} \mathrm{Li}=0.081\right)$ was adopted in the computations of our synthetic spectra. Atomic line data were taken from the VALD database (Kupka et al. 2000).

As mentioned above, the $\mathrm{TiO}$ veiling effect in the EK Boo spectrum is significant. This makes it difficult to place the continuum. For the local continuum, we adopted the highest point of the spectrum near the Li I line, at a wavelength of $6710.7 \AA$. Both the observed and synthetic spectra around $6708 \AA$ line are shown in Fig. 6.

We cannot find any traceable presence of the Li I $6708 \AA$ resonance line in the spectrum of EK Boo. Using alternative synthetic spectra, an upper limit to the lithium abundance of $\log \varepsilon(\mathrm{Li}) \leq-0.8$ was determined.

\section{Mass and evolutionary status of EK Boo}

In Sect. 3.3 we derive an effective temperature of $3400 \mathrm{~K}$ for EK Boo. In the literature, values of $3420 \mathrm{~K}$ and $3577 \mathrm{~K}$ are given, according to Perrin et al. (1998) and Dyck et al. (1998), respectively. Hence, we here determine the mass and evolutionary

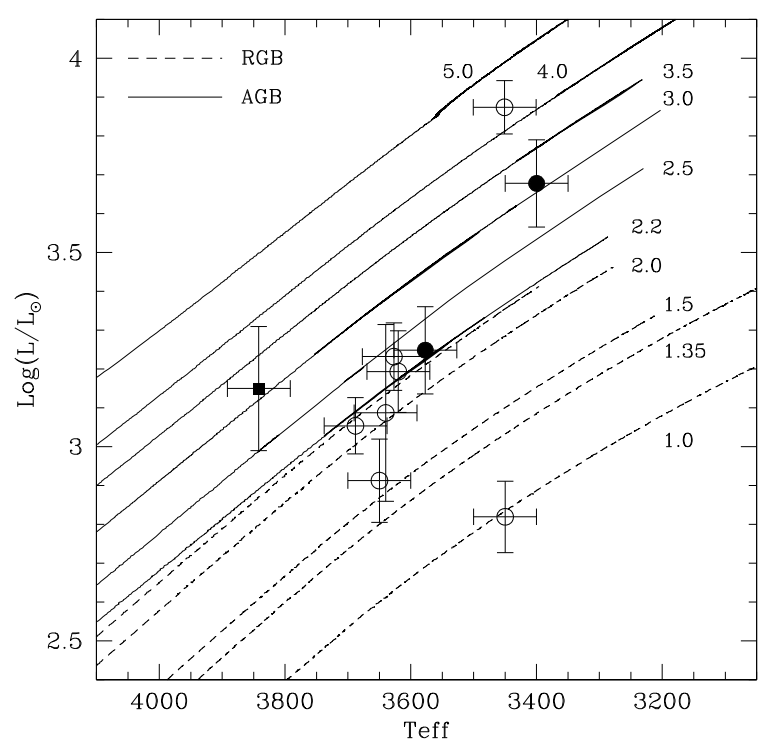

Fig. 7. Position in the H-R diagram of EK Boo and other M giants, adopting $T_{\text {eff }}$ from the literature, parallaxes from the New Reduction Hipparcos catalogue by van Leeuwen (2007), $V$ magnitudes from the 1997 Hipparcos catalogue, and bolometric corrections from Buzzoni et al. (2010). EK Boo's position (filled circles) is shown for two different effective temperatures ( $3420 \mathrm{~K}$ and $3577 \mathrm{~K}$, see text). $\beta$ And, another Zeeman detected $\mathrm{M}$ giant, is represented by a filled square. Standard evolutionary tracks for solar composition and various initial stellar masses (as indicated) are also plotted (Lagarde \& Charbonnel, in prep.). Dotted lines show the evolution up to the tip of the first ascent (the RGB) for the low-mass models (1.0 to $2.2 M_{\odot}$ ), while AGBs are indicated by solid lines for the intermediate-mass stars $\left(2.2 M_{\odot}\right.$ and above). For clarity, only the $2.2 M_{\odot}$ model is shown with both RGB and AGB.

status of EK Boo on the basis of both these effective temperatures. We use the parallax from the New Reduction Hipparcos catalogue by van Leeuwen (2007), the $V$ magnitude from the 1997 Hipparcos catalogue, and bolometric corrections according to Buzzoni et al. (2010).

Figure 7 shows the positions of EK Boo (black circles, for the two $T_{\text {eff }}$ quoted above) and other M giants (see Table 4) in the HR diagram, in comparison to standard stellar evolutionary tracks. These have been computed with the code STAREVOL by Lagarde \& Charbonnel (in prep.) for solar metallicity (with Asplund et al. 2005, chemical composition), ignoring stellar rotation. Tracks are shown for models with masses between 1.0 and $5.0 M_{\odot}$.

We focus here on the luminosity and effective temperature range of our sample stars, so that only the relevant RGB and AGB sequences (dashed and solid lines, respectively) appear on the graph. While low-mass stars $\left(\leq 2.2 M_{\odot}\right)$ ignite He-burning in a degenerate core at the tip of the RGB, i.e., at very high luminosity and cool effective temperature, more massive stars start central-He burning at much lower luminosities and hotter $T_{\text {eff. }}$. Hence, the short RGB-tracks of the latter fall outside Fig. 7, showing only their AGBs. Respectively, for the low-mass stars we have plotted only the RGB sequences. As an exception, the 2.2 $M_{\odot}$ model is shown with both RGB and AGB.

When an effective temperature of EK Boo of $3400 \mathrm{~K}$ is considered, this comparison leads to an estimate of the mass of $3.1 \pm 0.5 M_{\odot}$. The uncertainty quoted here reflects only the parallax error. And in this case, EK Boo is found to be evolving 
already on the AGB. At the luminosity of EK Boo our $3 M_{\odot}$ model has just undergone a first and relatively weak thermal pulse. By contrast, an alternative effective temperature of $3577 \mathrm{~K}$ would lead to a lower initial mass of $2.3 \pm 0.3 M_{\odot}$, when compared to the tracks. Then the star would be either close to the tip of the first ascend (i.e., on the red giant branch, RGB), or on the AGB near the onset of the thermal pulse phase. In these two cases (i.e., 3.1 or $2.3 M_{\odot}$, for $T_{\text {eff }}=3400$ or $3577 \mathrm{~K}$, respectively), the corresponding stellar radii are 200 and $109 R_{\odot}$, respectively if one uses the 2007 parallax of EK Boo.

According to Aurière et al. (2009, see their Fig. 5), consideration of stellar rotation would neither change the derived mass value, nor the evolutionary status for such a bright giant. Let us point out, however, that the models presented here were computed without core overshooting. For a given stellar mass, introducing core overshooting actually leads to a more massive $\mathrm{He}$-core at the end of the main sequence, and thus to He-ignition at a somewhat lower RGB luminosity. In that case, the RGB tipluminosities for stars above $\sim 1.5 M_{\odot}$ could actually be lower than those shown in Fig. 7. This makes an even stronger case for EK Boo being an AGB star.

Major uncertainties on the determination of the mass and evolutionary status of our other sample stars actually come from the errors on the effective temperature and on the parallax of the star, as can be seen in Fig. 7. However, uncertainties of the bolometric corrections are also significant. Here, we use the prescription by Buzzoni et al. (2010), who provide a fitting function for $B C_{V}$ vs. $T_{\text {eff }}$, supposed to be valid in the range of $3300 \leq T_{\text {eff }} \leq 5000 \mathrm{~K}$. For $T_{\text {eff }}=3400 \mathrm{~K}$, as derived above for EK Boo, this gives $B C_{V}=-3.255$.

Buzzoni et al. (2010) show that for stars with $T_{\text {eff }} \geq 4000 \mathrm{~K}$ the $B C_{V}-T_{\text {eff }}$ relation does not depend on stellar metallicity as far as atomic transitions prevail as the main source of metal opacity in their spectra. However, for a star as cool as $T_{\text {eff }}=3400 \mathrm{~K}$, molecular opacity (mainly due to TiO) becomes important in visual wavelength, which could in turn affect the derived $B C_{V}$ value. To the cool end $\left(T_{\text {eff }} \leq 3700 \mathrm{~K}\right)$, the sample used by Buzzoni et al. (2010) is actually biased in this respect, since only giants from a metal-rich open cluster (NGC 6791, $[\mathrm{Fe} / \mathrm{H}]=+0.4)$ were available for the bolometric correction determination.

Hence, we compared the Buzzoni-values of $B C_{V}$ with those which would follow for our M giants from Flower (1996) or Bessel et al. (1998). For less cool stars, i.e. $T_{\text {eff }} \geq 3500 \mathrm{~K}$, all these bolometric corrections do not differ significantly from each other, but for cooler stars they do. For $T_{\text {eff }}=3400 \mathrm{~K}$, as derived for EK Boo, we get a $B C_{V}=-2.70$ according to Flower (1996), and -3.07 when using Bessel et al. (1998). Taking into account these significant uncertainties in $\mathrm{BC}$, the best we can conclude is that the mass of EK Boo lies in the range of 2.0-3.6 $M_{\odot}$.

The absence of the ${ }^{7} \mathrm{Li}$ enrichment in the atmosphere of EK Boo is consistent with what is expected for a star within this mass range and at this evolutionary stage. The star has indeed depleted in the previous phases of its evolution all the ${ }^{7} \mathrm{Li}$ it was born with, and it is not massive nor bright enough to produce fresh ${ }^{7} \mathrm{Li}$ via the so-called Cameron \& Fowler (1971) mechanism on the TP-AGB (see e.g. Smith 2010, and reference therein) ${ }^{2}$.

\footnotetext{
2 Sackmann \& Boothroyd (1992) predict that Li-rich AGB stars will have a mass range of 5 to $7 M_{\odot}$ for a solar metallicity.
}

\section{Rotation and dynamo operation in EK Boo}

\subsection{Rotation}

As for most $M$ giants, the rotational period of EK Boo is not known. But in this case, $v \sin i$ has been determined. The CORAVEL value obtained on the basis of the cross-correllation method (Melo et al. 2001) is $11 \pm 1 \mathrm{~km} \mathrm{~s}^{-1}$ (Hünsch et al. 2004). Our NARVAL data and the spectral synthesis method (permitting us to account for rotational and macroturbulent broadening) yield $v \sin i$ of $8.5 \mathrm{~km} \mathrm{~s}^{-1}$ with an accuracy $0.5 \mathrm{~km} \mathrm{~s}^{-1}$. To estimate the projected rotational velocity, several synthetic spectra were generated by MOOG with different values of $v \sin i$ and $v_{\text {macro. }}$. The best fit was found for the following combination of the line-broadening factors: $v \sin i=8.5 \mathrm{~km} \mathrm{~s}^{-1}$ and $v_{\text {macro }}=2.0 \mathrm{~km} \mathrm{~s}^{-1}$. An adoption of low projected rotation velocity combined with high macroturbulent velocity leads to synthetic spectra that are noticeably different from the observed one. However, we have to mention that we do not know the real geometry of macroturbulent motions in the atmosphere of EK Boo. In our calculations we used an assumption of radial-tangential macroturbulence profile (Gray 1992).

The difference between these two values could simply arise because of the different methods of $v \sin i$ determination. In general, $\mathrm{M}$ giants as very evolved stars are expected to be slow rotators. In this respect, both values above are surprisingly high! Hence, EK Boo appears to be a single $M$ giant with a relatively fast rotation, compared to the statistical value for its spectral class (Zamanov et al. 2008).

An estimate of the rotational period $P$ of the star (upper limit only) could be made using the radius of the star, as derived from its angular diameter. Adopting $\theta$ of $7.90 \pm 0.40$ mas (Dyck et al. 1998), as well as a distance of $248 \pm 25$ pc (by the new Hipparcos parallaxes of van Leeuwen 2007), we obtain a radius of $210 \pm 21 R_{\odot}$. This value is about twice as high as the statistical value for an M 5 giant $\left(120 R_{\odot}\right)$ given in Dumm \& Schild (1998). It is however consistent with the value of $200 R_{\odot}$ derived in Sect. 4 for EK Boo being an AGB star with a $T_{\text {eff }}$ of $3400 \mathrm{~K}$. Assuming rigid body rotation and $v \sin i$ of $8.5 \mathrm{~km} \mathrm{~s}^{-1}$, we obtain $P \leq 1247^{\mathrm{d}}$ for the empirical radius of $210 R_{\odot}$, and $P \leq 714^{\mathrm{d}}$ for the statistical radius of $120 R_{\odot}$. For $v \sin i=11 \mathrm{~km} \mathrm{~s}^{-1}$, these values are $964^{\mathrm{d}}$ and $552^{\mathrm{d}}$, respectively.

Of course, any tilt of the giant's axis into the line of sight would in deed mean it is rotating even faster than suggested by the radial velocity measurement. Adopting the statistical value of $\sin i=\pi / 4$ and the empirical radius, we then obtain a period of $846^{\mathrm{d}}$ for $v \sin i=8.5 \mathrm{~km} \mathrm{~s}^{-1}$ (or $654^{\mathrm{d}}$ for $v \sin i=11 \mathrm{~km} \mathrm{~s}^{-1}$ ).

In future, our observations and Zeeman Doppler Imaging (ZDI) method will be able to shed more light on the actual rotational period of EK Boo.

\subsection{Dynamo operation and its origin}

Indirect evidence for the magnetic activity of EK Boo has been reported before our NARVAL study. The star attracted the attention of Hünsch et al. (1998) because of its high X-ray luminosity. Later, Hünsch et al. (2004) studied its variability in X-ray and optical activity indicators. They too ruled out the possibility of a symbiotic nature of EK Boo.

Our study (see Sect. 3.1) revealed a variable longitudinal magnetic field in EK Boo for which a dynamo could be a possible explanation. A dipole (as a remnant of an Ap star on the main sequence) is an unlikely explanation, considering the big radius and big convective envelope at this evolutionary stage. We now 
attend to the question about the possible origin of the dynamo and its type in this evolved star.

A star of about 2.2-3.6 $M_{\odot}$ at the evolutionary stage of EK Boo has just entered the thermally-pulsing phase on the AGB. It has a complex internal structure with an O/C core surrounded by He- and H-burning shells, and a very deep and extended convective envelope.

Turbulent motions and fast rotation could result in dynamo action of the $\alpha-\omega$ type, as described in Nordhaus et al. (2008). Could such a dynamo explain the magnetic field in EK Boo? We calculated the Rossby number $\left(R_{\mathrm{O}}\right)$, which is indicative for an efficient dynamo action of this type. For the statistical rotational period of $846 \mathrm{~d}$ (see Sect. 5.1) and a convective turnover time $\tau_{\mathrm{c}}$ of $182 \mathrm{~d}$, as obtained from the model of $2.5 M_{\odot}$ described in Sect. 4, we determined a $R_{\mathrm{O}}$ of about 4.7. For a period of $654 \mathrm{~d}$ (as for a smaller, statistical radius, see Sect. 5.1), the value of $R_{\mathrm{o}}$ would be 3.6. For these values, we cannot expect a very efficient $\alpha-\omega$ dynamo for EK Boo, but this dynamo might be still operationable (see Soker \& Tylenda 2007). Our future observations and a direct determination of the rotational period could lead to a better knowledge of the Rossby number and the $\alpha-\omega$ dynamo efficiency in this $\mathrm{M}$ giant.

Considering the high density and height difference between the photospheric layers of the giant and its bottom of the convection zone deep inside, it is well possible that the deep layers rotate a lot faster then the surface, which suffers from magnetic braking. In this way, the $\alpha-\omega$ dynamo may yet be more efficient than expected on the basis of the surface rotational rate.

The question remains of where the required angular momentum should come from to run this dynamo. Schröder et al. (1998) studied the evolutionary status of evolved stars that have coronal X-ray emission, which is a good indicator of stellar activity among less cool giants. And Konstantinova-Antova et al. (2009) studied fast rotating single $\mathrm{G}$ and $\mathrm{K}$ giants with detected magnetic field. The most active giants in their samples are above $1.5 M_{\odot}$, at different evolutionary stages after the main sequence (MS): first gap crossers, stars near the base of the RGB and its lower part. These stars had no convective outer layers and, consequently, no stellar activity on the MS. Hence, magnetic braking cannot yet have consumed their fast rotation and fresh activity has set in at these early post-MS stages. By contrast, EK Boo has evolved much further and its convective envelope is a lot more extended. It should have slowed down long ago, and fresh angular momentum is required here to run its faster rotation and a dynamo.

The possible source is the contracting core region of this giant: without any angular momentum transfer, the contracting core would be considerably speeding up its rotation rate, both in an RGB and an AGB giant of such a luminosity such as EK Boo. Its final product, a white dwarf, would have to rotate as fast as in minutes, which is physically impossible. Instead, we now have even direct evidence (see Charpinet et al. 2009) that white dwarfs are rotating a lot slower, with periods of the order of a day. Hence, evidently over $99 \%$ of the angular momentum of the core region is lost during the late evolutionary stages. As discussed by Talon \& Charbonnel (2008), internal gravity waves may strongly contribute to the angular momentum redistribution in intermediate-mass stars such as EK Boo, when they reach the early-AGB phase. Hence, it may serve the expanded convective envelope to keep it spinning at a sufficient rate to run a dynamo process in $\mathrm{M}$ giants.

The other possible dynamo type operating in AGB giants is the turbulent dynamo, where the turbulent motions play the main role in the field amplification process and in the restoration of the poloidal component (Brandenburg 2001). However, for EK Boo this dynamo is less efficient than the $\alpha-\omega$ one. Soker \& Zoabi (2002) estimated that in AGB stars the magnetic field can reach only values of less than $1 \mathrm{G}$. This is inconsistent with the much larger net field strength, which we observe in EK Boo.

A second explanation for the magnetic field in this $M$ giant could be given if EK Boo were a merger of a binary system, which would now be in its spin-down phase. Then an $\alpha-\omega$ dynamo is well possible to operate (Soker \& Tylenda 2007). The large radius of EK Boo together with the high $v \sin i$ appears to support this idea. But for the moment, the accuracy of the angular diameter measurement and of the parallax are not high enough to give a reliable determination of the radius of the star. In future, we will need more precise values for these parameters, to be able to distinguish between these two possibilities for $\alpha-\omega$ dynamo operation in the star.

Another possibility for spinning up a giant and to induce dynamo action is planet engulfment (Siess \& Livio 1999). In this case, according to Melo et al. (2005), there should be an enhanced Li abundance. Indeed, Drake et al. (2002) found that among the rapid rotating $\left(v \sin i \geq 8 \mathrm{~km} \mathrm{~s}^{-1}\right.$ ) single $\mathrm{K}$ giants, there is a very large proportion $(\sim 50 \%)$ of Li-rich objects. By contrast, there is only a very small fraction $(\sim 2 \%)$ of Li-rich stars among the common type of slowly rotating $\mathrm{K}$ giants.

Unfortunately, we do not observe any enhanced lithium abundance in EK Boo, although we analysed this point carefully (see Sect. 3.4). However, note that only a recently engulfed big planet would cause a significant $\mathrm{Li}$ content on the star, since $\mathrm{Li}$ is a fragile element. The spin-down time, on the other hand, remains unclear, but it might exceed the lithium life-time. Hence, we cannot completely exclude the possibility that the enhanced rotation might be a result of a big planet engulfment.

\section{Are there other magnetically active $M$ giants?}

If dynamo operation on the AGB is a result of the evolution of the star rather than an exotic case like binary merging or planet engulfment, we should expect more giants to have a magnetic field at the stage of EK Boo. As mentioned already in Sect. 1, there is indirect evidence for such activity, which stimulated us to begin observing several other $\mathrm{M}$ giants of interest in that respect.

Hence, we observed eight other M giants with the same procedures as for EK Boo (see Sect. 2). Three of them are known for their high X-ray emission: 15 Tri, 42 Her, and HD $187372=$ HR 7547 (Hünsch et al. 1998, 2004). The sources 15 Tri and HD 187372 are also known as spectroscopic binaries of a long period (i.e., no synchronization), and $42 \mathrm{Her}$ is considered to have a stable $R V$ (Hünsch et al. 2004; Famaey et al. 2009). Our own NARVAL $R V$ measurements confirm these findings (the specific case of EK Boo is discussed in Sect. 3.2). Five other $\mathrm{M}$ giants are fast rotators (Zamanov et al. 2008). These are HD 167006, HD 184786, 8 And = HD 219734, HD 18191, and $\beta$ And $=$ HD 6860. All these giants show no radial velocity variations and may be considered to be single stars (Famaey et al. 2005).

Data of all observed giants are presented in Table 4. The effective temperatures are from the VizieR catalogues. The X-ray luminosity $L_{\mathrm{x}}$ is from Hünsch et al. (2004); the measurements are both from the ROSAT and Einstein satellites. Positions in the HR diagram are shown in Fig. 7, assuming parallaxes from the New Reduction Hipparcos catalogue by van Leeuwen (2007), V magnitudes from the 1997 Hipparcos catalogue, and bolometric corrections from Buzzoni et al. (2010). 
Table 4. Data for the observed M giants.

\begin{tabular}{|c|c|c|c|c|c|c|c|c|c|c|}
\hline Star & Other name & Sp class & $\begin{array}{c}T_{\text {eff }} \\
\mathrm{K}\end{array}$ & $\begin{array}{c}v \sin i \\
\mathrm{~km} \mathrm{~s}^{-1}\end{array}$ & $\log L_{\mathrm{x}}$ & Date & No. exp. & Detection & $\begin{array}{l}B_{1} \\
\mathrm{G}\end{array}$ & $\begin{array}{l}\sigma \\
\mathrm{G}\end{array}$ \\
\hline HD 130144 & EK Boo & M 5III & 3400 & $8.5 / 11$ & $30.30-31.15$ & See Table 1 & & $\overline{\mathrm{DD}}$ & & \\
\hline HD 6860 & $\beta$ And & M0III & 3842 & 5.6 & & $\begin{array}{c}16+26 \text { Sep. } 08 \\
24 \text { Sep. } 09\end{array}$ & $\begin{array}{l}14 \\
16\end{array}$ & $\begin{array}{c}\text { MD } \\
\text { nd }\end{array}$ & $\begin{array}{l}-0.95 \\
-0.29\end{array}$ & $\begin{array}{l}0.16 \\
0.10\end{array}$ \\
\hline HD 16058 & 15 Tri & M3III & 3640 & 5.4 & 30.8 & $\begin{array}{c}20+21 \text { Sep. } 08 \\
20 \text { Dec. } 08\end{array}$ & $\begin{array}{l}5 \\
3\end{array}$ & $\begin{array}{l}\text { nd } \\
\text { nd }\end{array}$ & $\begin{array}{l}-0.68 \\
-1.05\end{array}$ & $\begin{array}{l}0.38 \\
0.59\end{array}$ \\
\hline HD 18191 & RZ Ari & M6III & 3450 & 9.6 & & $16+21$ Sep. 08 & 5 & nd & -0.89 & 0.45 \\
\hline HD 150450 & 42 Her & M2.5III & 3650 & 2.5 & 29.41 & $\begin{array}{c}19+30 \text { Sep. } 08 \\
25 \text { Feb. } 09\end{array}$ & $\begin{array}{l}8 \\
4\end{array}$ & $\begin{array}{l}\text { nd } \\
\text { nd }\end{array}$ & $\begin{array}{l}-0.37 \\
-0.01\end{array}$ & $\begin{array}{l}0.19 \\
0.31\end{array}$ \\
\hline HD 167006 & V669 Her & M3III & 3688 & 5.2 & & $\begin{array}{c}16+21 \text { Sep. } 08 \\
21 \text { May } 09\end{array}$ & $\begin{array}{l}12 \\
16\end{array}$ & $\begin{array}{l}\text { nd } \\
\text { nd }\end{array}$ & $\begin{array}{c}-0.85 \\
0.97\end{array}$ & $\begin{array}{l}0.32 \\
0.39\end{array}$ \\
\hline HD 184786 & V1743 Cyg & M4.5III & 3451 & 7.8 & & $15+25$ Sep. 08 & 8 & nd & -0.16 & 0.34 \\
\hline HD 187372 & HR7547 & M1III & 3620 & 4.4 & 30.64 & $\begin{array}{c}19+29 \text { Sep. } 08 \\
25 \text { Feb. } 09 \\
24 \text { Nov } 09\end{array}$ & $\begin{array}{l}8 \\
4 \\
3\end{array}$ & nd & $\begin{array}{c}0.31 \\
-0.24 \\
-0.82\end{array}$ & $\begin{array}{l}0.34 \\
0.47 \\
0.53\end{array}$ \\
\hline HD 219734 & 8 And & M2III & 3627 & 4.9 & & $15+30$ Sep. 08 & 8 & nd & -0.28 & 0.30 \\
\hline
\end{tabular}

Notes. Individual columns list HD number, name of the star, spectral class, effective temperature $T_{\text {eff }}$ from VizieR catalogues, $v \sin i$, $\log$ of X-ray luminosity (in $\mathrm{erg} \mathrm{s}^{-1}$ ), detection $(\mathrm{DD}=$ definite, $\mathrm{MD}=$ marginal, $\mathrm{nd}=\mathrm{no}), B_{1}$ and its error in $\mathrm{G}$.

All these are stars in the mass interval 1.0-4.5 $M_{\odot}$, situated near the tip of the RGB or on the AGB. The least cool member of the sample, $\beta$ And is among the more massive stars in our study $\left(3.1 \pm 0.5 M_{\odot}\right)$, and is located on the AGB. Note that at the adopted $T_{\text {eff }}$ of $\beta$ And, the uncertainty on the $B C_{V}$ is much smaller than for EK Boo. Consequently, the uncertainty of its mass is mainly owing to the error of the Hipparcos parallax.

With the exeption of $\beta$ And, which is much brighter, these $\mathrm{M}$ giants are in the same range of magnitude as EK Boo. For these reasons, their magnetic field detection and $B_{1}$ measurements are of a similar accuracy as those of EK Boo. The three fast rotators (HD 18191, HD 184786, HD 219734) were observed only in one month (September 2008). The sources $\beta$ And and HD 167006 were observed during two months. The three X-ray luminous giants were observed during two or three months: September and December 2008 (15 Tri), September 2008 and February 2009 (42 Her), and HD 187372 was observed in September 2008, February 2009, and November 2009.

A magnetic field was not detected with certainty for any of these stars within the limit of our accuracy $(0.5 \mathrm{G})$. However, for $\beta$ And, the brightest star in this sample, we obtained a marginal detection of magnetic field (see Fig. 8) in September 2008, after averaging 14 spectra. The measured $B_{1}$ is $-0.95 \pm 0.2 \mathrm{G}$. New observations were obtained on 24 September 2009: a weak Stokes $V$ signal is still visible, but at a non significant LSD statistical level (Donati et al. 1997).

This means, that magnetic fields may well be present in these stars, but with a weaker averaged longitudinal field, wherefore a different observational strategy might be necessary. However, a weak field seems an unlikely explanation for 15 Tri and HD 187372, which are X-ray sources as luminous as EK Boo, but which have to date not yet yielded any Zeeman detection of a mean magnetic field. As suggested in Sect. 3.1, magnetic areas with opposite polarities on the surfaces of these stars could produce averaged longitudinal magnetic fields close to zero, and that possibly for extended periods. Actually, EK Boo displays big differences in its magnetic field behaviour over an extended time interval (e.g. April-December 2008). Hence, we will need to monitor all suspected $\mathrm{M}$ giant stars both deeper and for longer periods of time.

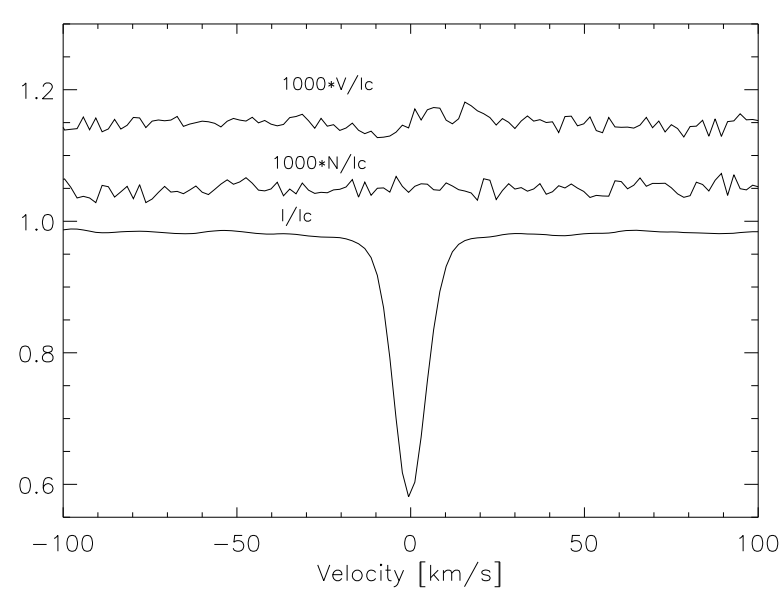

Fig. 8. Averaged Stokes $I$ (bottom), $N$ (middle) and $V$ (upper) profiles for $\beta$ And. The spectra were obtained in September 2008.

For the X-ray luminous M giants, it may not entirely be excluded that a secondary star is the origin the X-ray radiation. Two of them, 15 Tri and HD 187372 are long-period spectroscopic binaries (Hünsch et al. 2004; Famaey et al. 2009), and 42 Her is a visual binary. Their $v \sin i$, however, are smaller than the value of EK Boo, which could simply mean that they are less active. All these stars have Ca II emission, but their $\mathrm{K}$ emission cores show only little variability, much less than what we observe in EK Boo. This may support the idea that there is a link between their lower rotation velocity and a lower activity level in these three $\mathrm{M}$ giants.

The five fast rotating single $\mathrm{M}$ giants also exhibit Ca II emission cores with a different strength. But we still lack sufficient observational data of them to judge their variability. In any case, all M giants (with one exception: HD 18191) have lower $v \sin i$ values than EK Boo and a lower activity level could not be excluded, too.

More observations will answer the question of magnetic field in these $\mathrm{M}$ giants. In the future, we also want to expand our sample of $\mathrm{M}$ giants with some Mira variables, for which magnetic 
field detection has been reported from maser emission lines (Herpin et al. 2006). In this way, we will be able to draw a more complete picture of magnetic activity in AGB stars, and we will finally know, whether EK Boo is a special case, or rather whether this M giant is just the "tip of an iceberg" of magnetically active AGB stars.

\section{Conclusions}

1. The M 5 giant EK Boo was observed with NARVAL in the period of April 2008 to March 2009 during 10 nights. We analysed the $R V$ behaviour of the star and came to the conclusion that EK Boo is likely a single star, and that the observed variations of its $R V$ are due to some kind of pulsation. Its position on the $\mathrm{H}-\mathrm{R}$ diagram reveals that EK Boo is of 2.0-3.6 $M_{\odot}$, located on the AGB or near the tip of the RGB.

2. Magnetic field was definitely detected in the photosphere of EK Boo and its longitudinal component $B_{1}$ was of the order of few Gauss. It is of a variable strength, and the activity indicators $\mathrm{Ca}$ II $\mathrm{K} \& \mathrm{H}$ also show variability. EK Boo is the first $\mathrm{M}$ giant for which surface magnetic field is detected.

3. In March 2009, a complex structure in Stokes $V$ was observed. This find may be indicative of a dynamo operation. In that case, EK Boo could be the first M giant with proof of dynamo operation.

4. A dynamo of the $\alpha-\omega$ type is more likely to operate there than a turbulent dynamo. The driving mechanism for the $\alpha-\omega$ dynamo operation could be angular momentum dredgeup from the interior, binary merging, or perhaps a planet engulfment, but the absence of a high Li abundance in EK Boo speaks against the latter (unless the spin-down time is significantly longer than the Li destruction time). A turbulent dynamo appears to be challenged by the high measured field strength of EK Boo.

5. For one more $\mathbf{M}$ giant, $\beta$ And, we got a marginal detection of the magnetic field with a $B_{1}$ strength of $-0.95 \mathrm{G}$. A different observational strategy is required to be able to detect a weaker magnetic field in our other sample $\mathrm{M}$ giants, and to answer the question, what kind of a special case EK Boo is.

Note added in proof. After this paper was accepted for publication, we obtained new spectra for some of the M giants of our sample. Two stars, V669 Her and RZ Ari are definitively Zeeman detected, and their longitudinal magnetic field is about $1 \mathrm{G}$ and $3 \mathrm{G}$, respectively.

Acknowledgements. We thank the TBL team for their service observing support. Our observation time was granted under an OPTICON programme. We further acknowledge use of the Hipparcos database, the VizieR catalogue access tool (CDS, Strasbourg, France) and of the VALD data base in Vienna. R.K.-A. and I.S. acknowledge partial financial support under Bulgarian NSF contract DO 02-85/2009. R.K.-A. appreciates the possibility to work in LATT - Tarbes as an invited researcher in the spring 2009. C.C. acknowledges financial support from the French Programme National de Physique Stellaire (PNPS) from CNRS/INSU and from the Swiss National Science Foundation (FNS).

\section{References}

Adelman, S. J. 2001, BaltA, 10, 589

Anders, E., \& Grevesse, N. 1989, Geochim. Cosmochim. Acta, 53, 197
Aurière, M. 2003, in Magnetism and Activity of the Sun and Stars, ed. J. Arnaud, \& N. Meunier, EAS Publ. Ser., 9, 105

Aurière, M., Konstantinova-Antova, R., Petit, P., et al. 2008, A\&A, 491, 499

Aurière, M., Wade, G. A., Konstantinova-Antova, R., et al. 2009, A\&A, 504, 231

Bessell, M. S., Castelli, F., \& Plez, B. 1998, A\&A, 333, 231

Birch, K. P., \& Downs, M. J. 1994, Metrologia, 31, 315

Blackman, E. G., Frank, A., et al. Nature, 409, 485

Boothroyd, A. I., \& Sackmann, I.-J. 1999, ApJ, 510, 232

Brandenburg, A. 2001, ApJ, 550, 824

Buzzoni, A., Patelli, L., Bellazzini, M., et al. 2010, MNRAS, 403, 1592

Cameron, A. G. W., \& Fowler, W. A. 1971, ApJ, 164, 111

Charbonnel, C. 1994, A\&A, 282, 811

Charpinet, S., Fontaine, G., \& Brassard, P. 2009, A\&A, 493, 595

Donati, J.-F., Semel, M., Carter, B. D., et al. 1997, MNRAS, 291, 658

Donati, J.-F., Catala, C., Landstreet, J., \& Petit, P. 2006, in Solar Polarization Workshop, ed. R. Casini, \& B. Lites, ASPC Ser., 358, 362

Drake, N. A., de la Reza, R., da Silva, L., \& Lambert, D. L. 2002, AJ, 123, 2703

Dumm, T., \& Schild, H. 1998, NewA, 3, 137

Dyck, H. M., van Belle, G. T., Thomson, R. R., et al. 1998, AJ, 116, 981

Famaey, B. 2005, Radial velocities for $6691 \mathrm{~K}$ and M giants, Catalogue (Famaey)

Flower, P. J. 1996, ApJ, 469, 355

García-Hernández, P., García-Lario, Plez, B., et al. 2007, A\&A, 462, 711

Gray, D. F. 1992, The observation and analysis of stellar photospheres (Cambridge University Press)

Gurtovenko, E. A., \& Kostyk, R. I. 1989, Fraunhofer Spectrum and System of Solar Oscillator Strenths, Naukova Dumka, Kiev

Gustafsson, B., Edvardsson, B., Eriksson, K., et al. 2008, A\&A, 486, 951

Herpin, F., Baudry, A., Thum, C., et al. 2006, A\&A, 450, 667

Hinkle, K. H., Lebzelter, T., Joyce, R. R., \& Fekel, F. C. 2002, AJ, 123, 1002

Hobbs, L. M., Thorburn, J. A., \& Rebull, L. M. 1999, ApJ, 523, 797

Hünsch, M., Schmitt, J. H. M. M., Schröder, K. P., et al. 1998, A\&A, 330, 225

Hünsch, M., Konstantinova-Antova, R., Schmitt, J. H. M., et al. 2004, in Proc. IAU Symp., ed. A. Dupree, \& A. Benz, 219, 223

Karovska, M., Schlegel, E., Hack, W., et al. 2005, ApJ, 623, L137

Kholopov, P. N., Samus, N. N., Frolov, M. S., et al. 1998, GCVS4, C

Konstantinova-Antova, R., Aurière, M., Iliev, I. Kh., et al. 2008a, A\&A, 480, 475

Konstantinova-Antova, R., Aurière, M., Alecian, E., et al. 2008b, in Exploring the Solar System and the Universe, ed. V. Mioc, C. Dumitrache, et al., AIP Conf. Proc., 1043, 405

Konstantinova-Antova, R., Aurière, M., Schröder, K.-P., \& Petit, P. 2009, in Cosmic Magnetic Fields: From Planets, to Stars and Galaxies, ed. K. G. Strassmeier, A. G. Kosovichev, \& J. E. Beckman, 433

Kupka, F., Ryabchikova, T. A., Piskunov, N. E., Stempels, H. C., \& Weiss, W. W. 2000, Baltic Astron., 9, 590

Lebzelter, T. 1999, A\&A, 351, 644

Lebzelter, T., Kiss, L. L., \& Hinkle, K. H. 2000, A\&A, 361, 167

Luck, R. E., \& Lambert, D. L. 1982, ApJ, 256, 189

Melo, C. H. F., Pasquini, L., \& De Medeiros, J. R. 2001, A\&A, 375, 851

Melo, C. H. F., de Laverny, P., Santos, N. C., et al. 2005, A\&A, 439, 227

Morton, D. C. 2000, ApJS, 130, 403

Moutou, C., Donati, J.-F., Savalle, R., et al. 2007, A\&A, 473, 651

Nordhaus, J., Busso, M., Wasserburg, J. G., et al. 2008, ApJ, 684, L29

Perrin, G., Coude du Foresto, V., Ridgway, S. T., et al. 1998, A\&A, 331, 619

Plez, B. 1990, Mem. Soc. Astron. Ital., 61, 765

Plez, B. 1998, A\&A, 337, 495

Plez, B., Smith, V. V., \& Lambert, D. L. 1993, ApJ, 418, 812

Rees, D. E., \& Semel, M. 1979, A\&A, 74, 1

Sackmann, I.-J., \& Boothroyd, A. I. 1999, ApJ, 510, 217

Schröder, K.-P., Hünsch, M., \& Schmitt, J. H. M. M. 1998, A\&A, 335, 591

Siess, L., \& Livio, M. 1999, MNRAS, 308, 1133

Smith, G. H., \& Lambert, D. L. 1990

Smith, G. H., \& Shetrone, M. D. 2000, PASP, 112, 1320

Smith, V. V. 2010, Light Elements in the Universe, ed. C. Charbonnel, et al. (CUP), IAU Symp., 268, in press

Sneden, C. 1973, Ph.D. Thesis, Univ. of Texas

Soker, N., \& Zoabi, E. 2002, MNRAS, 329, 204

Soker, N., \& Tylenda, R. 2007, MNRAS, 375, 909

Talon, S., \& Charbonnel, C. 2008, A\&A, 482, 597

Udry, S., Mayor, M., Andersen, J., et al. 1997, ESASP, 402, 693

van Leeuwen, F. 2007, A\&A, 474, 653

Zamanov, R. K., Bode, M. F., Melo, C. H. F., et al. 2008, MNRAS, 390, 377 